\title{
Planificación de operaciones de construcción
}

\section{Construction operations planning}

\author{
Mauricio Gutiérrez-Loria', Ismael Mora-Chavarría², \\ Jonathan Quirós-Campos ${ }^{3}$
}

Fecha de recepción: 5 de agosto de 2020 Fecha de aprobación: 10 de noviembre de 2020

Gutiérrez-Loria, M; Mora-Chavarría, I; Quirós-Campos, J. Planificación de operaciones de construcción. Tecnología en

Marcha. Vol. 34-4 Octubre-Diciembre 2021. Pág 175-191.

doi https://doi.org/10.18845/tm.v34i4.5155

1 SERVIPLOTTER S.A, Universidad Fidélitas, Costa Rica. Correo electrónico: planos.magulo@gmail.com (iD) https://orcid.org/0000-0002-3583-1715

2 Novatecnia, Universidad Fidélitas, Costa Rica. Correo electrónico: ismamora9412@gmail.com (iD) https://orcid.org/0000-0002-5677-7690

3 Acueductos y Alcantarillados, Universidad Fidélitas, Costa Rica. Correo electrónico:johnqc13@gmail.com 


\section{Palabras clave}

Instalaciones provisionales; reglamento de construcciones - INVU; planes de operaciones; diagramas de flujo; cartas de proceso; planificación de corto plazo; implementación del sistema.

\section{Resumen}

El presente artículo está basado en el Capítulo 4. Planificación de operaciones de construcción del libro Administración de Operaciones de Construcción del Ing. Alfredo Serpell Bley (Chile), tratando de actualizar y adaptar la información, así como tropicalizar, y ejemplificar a la realidad, que se vive en el sector construcción en Costa Rica. El Ingeniero Serpell indica que la planificación de operaciones: "depende de los administradores de obra y tiene la influencia más directa sobre la productividad. El contexto del estudio de la planificación de operaciones estará dentro del marco de la planificación global del proyecto, por lo que permanentemente se destacarán las interrelaciones entre la una y la otra y, en especial, el hecho de que la planificación de operaciones es un subproducto de la planificación general del contrato o proyecto." [1]. En el ámbito de la construcción se entiende por operación a: la actividad de trabajo ya sea colocación o instalación de un elemento de construcción. El Ingeniero Serpell menciona que un proceso es: "una colección de tareas relacionadas entre ellas por una estructura tecnológica y una secuencia. Finalmente, una tarea es el elemento de trabajo más básico de los procesos y operaciones." [1].

\section{Keywords}

Provisional facilities; construction regulations - INVU; operations plans; flowgrams; process letters; short time planning; implementation of system.

\section{Abstract}

This article is based on Chapter 4. Planning of construction operations of the book Construction Operations Administration of Alfredo Serpell Bley (Chile), updating and adapting the information, as well as tropicalizing, and exemplifying the reality that is lived in the construction sector in Costa Rica. Engineer Serpell focused on operations planning: "depends on the construction managers and has the most direct influence on productivity. The context of the study of operations planning will be within the framework of the overall planning of the project interrelationships between one and the other will be highlighted permanently, and especially the fact that operations planning is a by-product of general contract or project planning. " [1]. In the context of construction, operation means that work activity that results in the placement or installation of a definable building element, Engineer Serpell mentions that a process is: "a collection of tasks related to each other by a technological structure and a sequence. Finally, a task is the most basic work item in processes and operations." [1].

\section{Introducción}

La planificación es una herramienta fundamental para la toma de decisiones en la construcción, por lo tanto, para la administración de un proyecto. Sin planificación se transforma en una serie de cambios aleatorios de dirección.

En un proyecto de construcción el planeamiento está dividido en:

1. "Planificación preliminar, de carácter estratégico, cuyos objetivos básicos son determinar los costos para propuestas o estudios de factibilidad y servir de base para la planificación del contrato o proyecto. 
2. Planificación del contrato o proyecto, de carácter táctico, cuyo objetivo es obtener el plan definitivo para la ejecución del proyecto.

3. Planificación de operaciones: El objetivo de esta planificación detallada es lograr que para cada operación se use la secuencia y el método más económico posible, de acuerdo con la planificación general del proyecto. Esto significa pensar en los detalles de una tarea, planificarla y coordinarla antes de ejecutarla, anticipando interferencias, falta de recursos, etc." [2].

La planificación puede ser definida como una metodología, que se va a utilizar para el cumplimiento de un objetivo específico. Una buena planificación confirma que cada tarea sea ejecutada correctamente en el lugar apropiado y en el momento oportuno.

Otra función importante de la planificación es la de servir como base de referencia para el control. Control es el proceso de toma de decisiones sobre la base de la información respecto a la situación actual para actuar sobre el desarrollo futuro de una obra y asegurar así el cumplimiento de los objetivos planteados. La planificación permite una utilización eficiente de los recursos y fortalece la posición del administrador. Esto último debido a que pueden minimizar las influencias negativas y transferir responsabilidades directivas a otros.

\section{Materiales y métodos}

\section{Planificación de operaciones}

\section{Tipos de planes de operaciones}

Actualizando lo indicado por el Ingeniero Serpell en Costa Rica, los planes de operaciones representan las instalaciones previas y provisionales y cómo se deben de ejecutar. Algunos de los planes de operaciones son los siguientes:

1. Los croquis: Entregan detalles de constructivos y de montaje, esquemas de diversas actividades. Las instrucciones del trabajo se notifican a través de una serie de croquis que exponen todos los elementos notables, conjuntamente con su relación con otras instalaciones previas y provisionales.

2. Hojas de asignación de trabajos: Un plan de este tipo responde las incógnitas de un operario antes que él las haga. Una vez concluido el trabajo, estas hojas se aprovechan para llevar un registro de información para el maestro de obras y el jefe de cuadrilla. Al terminar el trabajo, el maestro de obras deberá conocer el día en que éste se ejecutó, su duración y código.

3. Planos de taller: Son planos complementarios para la ejecución de etapas de construcción que tienen una gran dimensión o son muy complejas. En estos planos, se reúne toda la información relevante de los planos de taller detallados de las estructuras, instalaciones eléctricas, plomería, etc. Se incluyen las listas de materiales y puntos de control y/o inspección.

4. Modelos a escala o maquetas: Ayuda a contar con una base tridimensional para la planificación y diseño de operaciones e instalaciones auxiliares para la construcción. Las principales aplicaciones son:

- Distribución en planta.

- Uso y ubicación de guías, equipos e instalaciones.

- Montajes complejos de estructuras. 
5. Diagramas de flujo: Son herramientas sobre la base de una simbología modelo, permiten registrar las diferentes tareas que realiza un recurso y los procesos a los que es sometido.

6. Sistemas computacionales: Estas herramientas mediante la simulación virtual gráfica en computadora ahora sistemas de metodología BIM, desarrolla el proceso de construcción del proyecto, creando modelos gráficos para la planificación y diseño de operaciones de instalaciones. Dos de los programas son SYNCHRO Pro y Navisworks, estos programas permiten simular el movimiento en una obra a través de un conjunto de instalaciones ingresadas al computador.

7. Modelos de operaciones: Los más aplicables a la construcción son los modelos de teoría de colas o fenómenos de espera, los modelos de transporte y asignación, y los modelos gráficos de simulación computacional.

8. La planificación de corto plazo: Abarcar en general, todos los tipos de planes de operaciones mencionados previamente.

Estos modelos o sistemas son muy útiles como instrumentos de comunicación, ya que concentran una gran cantidad de información. Además, durante y después de la ejecución de la obra, pueden ser usados como ayudas a la empresa y capacitación del personal.

\section{Esquema para la confección de un plan de operaciones}

En general, un plan de operaciones debiera contener los siguientes elementos:

a. Descripción y alcance del plan

- Hacer una lista del equipo y herramientas especiales que deberán ser usadas.

- Indicar y describir los materiales a ser usados.

- Describir la distribución de las áreas de trabajo.

b. Plan de trabajo

- Preparar los croquis necesarios.

- Enumerar el material a ser recibido y los criterios de almacenamiento.

- Describir, paso a paso, el procedimiento de utilización de la mano de obra, materiales, herramientas y equipos para llevar a cabo la tarea.

c. Inspección, ensayos y control de calidad

- Enumerar pasos aplicables de inspección y ensayos.

- Indicar fuentes de información, tablas, normas y referencias para mayores detalles sobre las técnicas, materiales y métodos.

Una ventaja adicional de los planes de operaciones es que permiten a las empresas que los utilizan contar con una información valiosa para ajustar sus futuras estimaciones de rendimientos, duraciones y costos. También pueden ser usados en la confección de futuros planes de operaciones y como ayuda para la capacitación del personal.

Herramientas de planificación de operaciones

\section{Cartas de proceso}

Son diagramas lineales de la secuencia establecida para la ejecución de una operación. Es posible tener una ayuda visual del método en su conjunto, sirviendo de base para futuros análisis en busca de posibles mejoramientos. 
Las cartas de proceso pueden ser de distintos tipos:

a. Carta de proceso de mano de obra.

b. Carta de proceso de materiales.

c. Carta de proceso de equipos.

Las cartas de proceso se realizan con 5 símbolos estándar, su interpretación se indica en el cuadro 1.

Cuadro 1. Simbología de cartas de procesos.

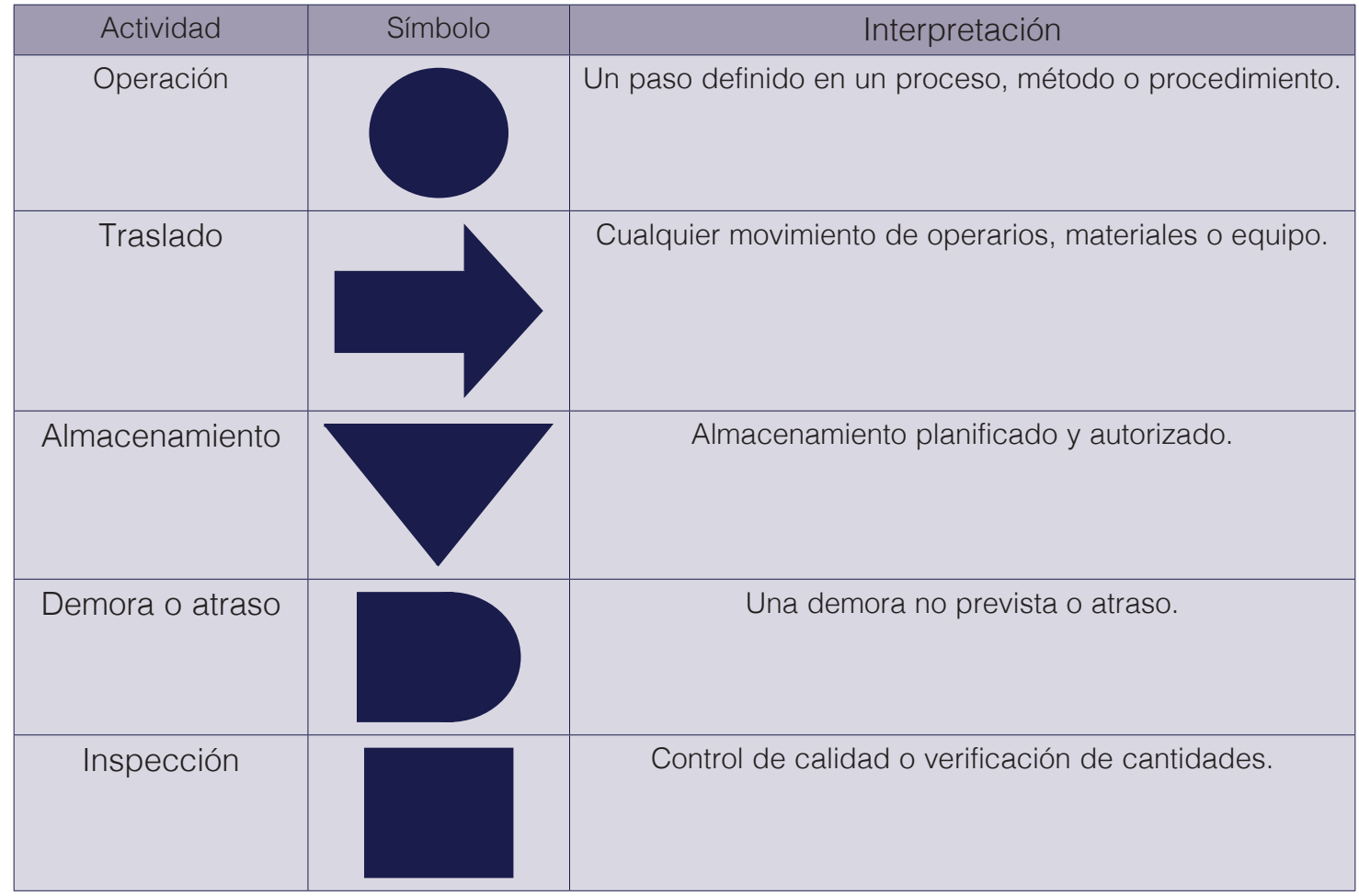

Fuente: [1]

En las cartas de proceso, los símbolos son conectados de forma que representen la secuencia de los eventos individuales de la operación que está en estudio.

\section{Diagramas de flujo}

Los diagramas de flujo corresponden a una representación gráfica y es uno de los métodos más comunes de representar procesos, gracias a su facilidad de construcción e interpretación. Sin embargo, es necesario tener en cuenta que existe una simbología asociada a ellos y que cada una representa una acción específica [3].

El diagrama de flujo de la figura 1 y la carta de proceso que se ilustra en la figura 2 corresponden a las actividades que realiza un operario. Es importante destacar que no se deben mezclar diferentes tipos de cartas de proceso, es decir, si lo que se analiza es el movimiento de un material, no se debe combinar con las actividades de otros recursos, tales como el personal o los equipos. 


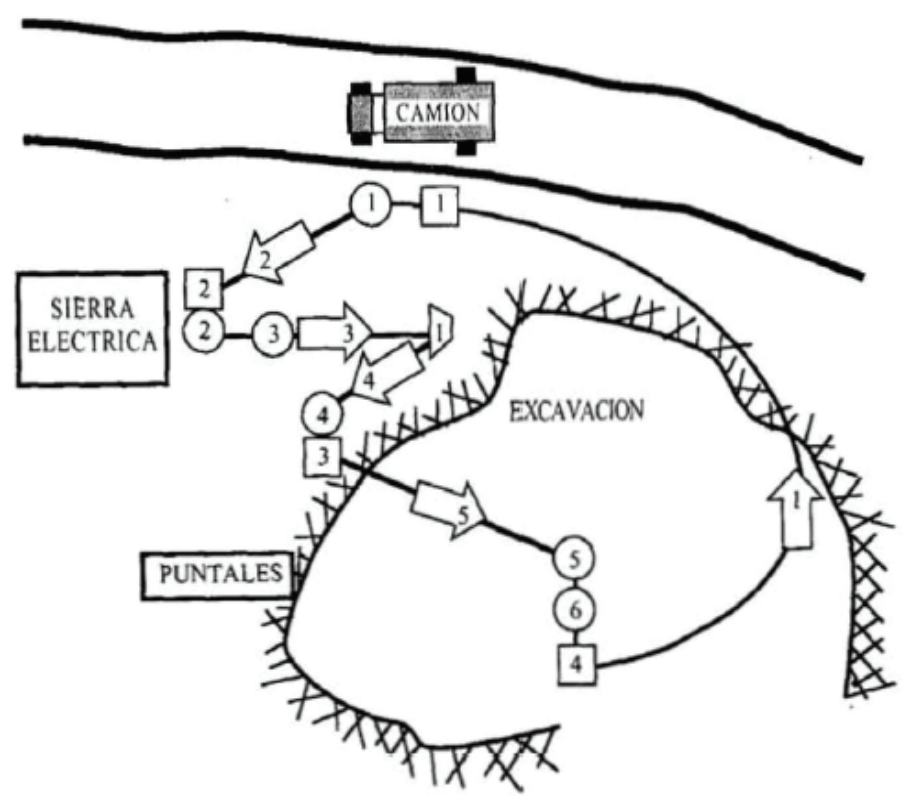

Figura 1. Ejemplo de diagrama de flujo. Fuente: [1].

La utilización de estos diagramas presenta varias ventajas en el proceso necesario para llevar a cabo una operación, entre estas ventajas el Mideplan menciona:

- "Favorecen la comprensión del proceso/procedimiento al mostrarlo como un dibujo. Esto por cuanto el cerebro humano reconoce más fácilmente los dibujos que la escritura en prosa. Un buen diagrama de flujo reemplaza varias páginas de texto.

- Permiten identificar los problemas y las oportunidades de mejora del proceso. Se identifican los pasos redundantes, los flujos de los reprocesos, los conflictos de autoridad, las responsabilidades, los cuellos de botella, y los puntos de decisión.

- Son una excelente herramienta para capacitar a los nuevos empleados y también a los que desarrollan la tarea, cuando se realizan mejoras en el proceso." [4].

La carta de proceso, en conjunto con el diagrama de flujo, pueden ser de mucha utilidad en el diseño de las instalaciones provisionales y en la distribución de las áreas de trabajo o diseño de un proyecto de construcción.

Análisis de procesos

En la planificación de operaciones, "una tarea importante es la relacionada con el análisis de los diferentes procesos que forman parte de las operaciones de construcción." [1]. Para lo cual se define el proceso como un sistema, por tanto, lo analizamos de la siguiente manera:

a. Definir límites y las interrelaciones externas.

b. Identificar recursos que participan en el proceso

c. Identificar el producto que resultara del proceso.

d. Comprender los flujos que existen en el sistema

e. Comprender el método de conversión del proceso. 


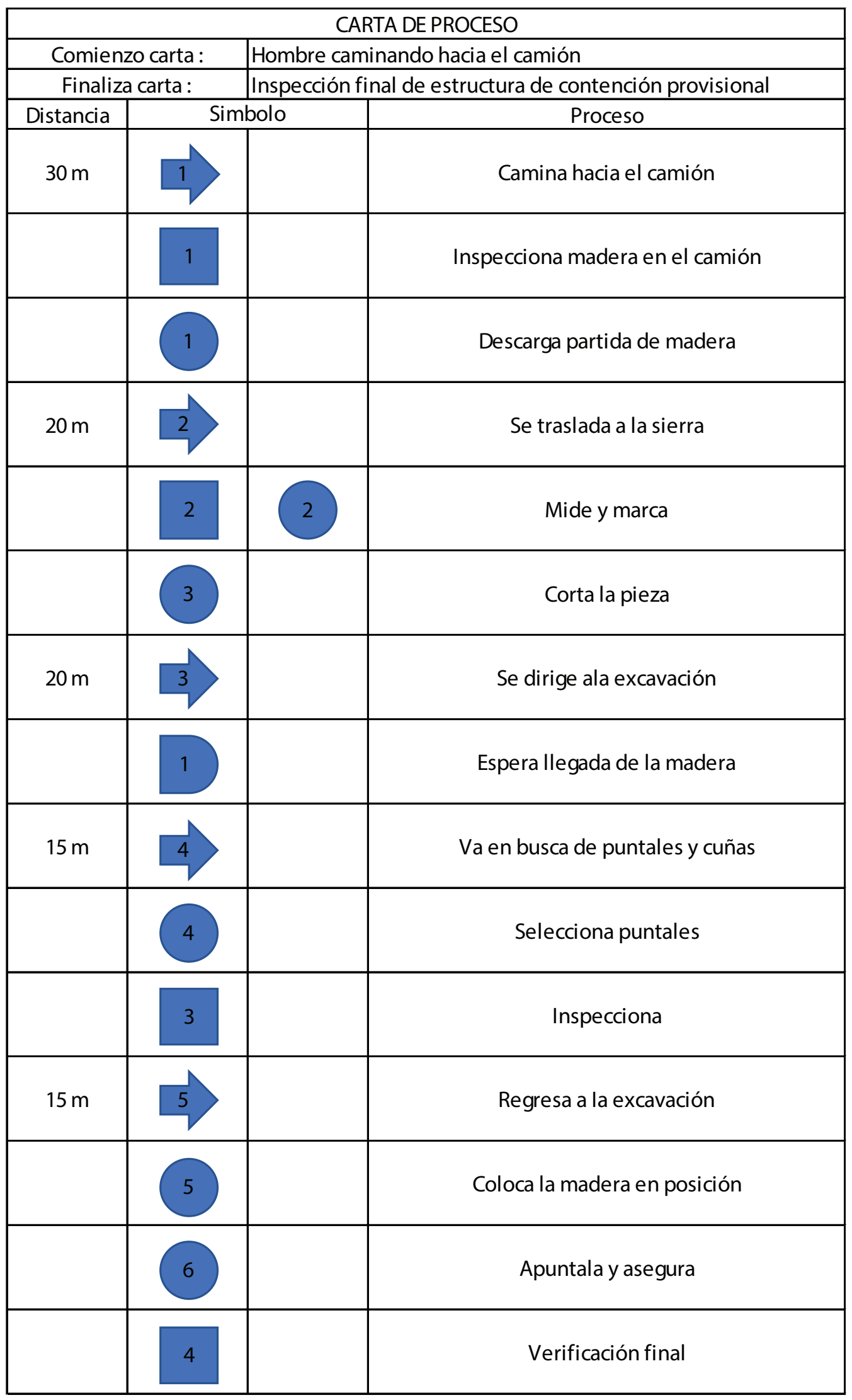




\section{Límite del sistema}

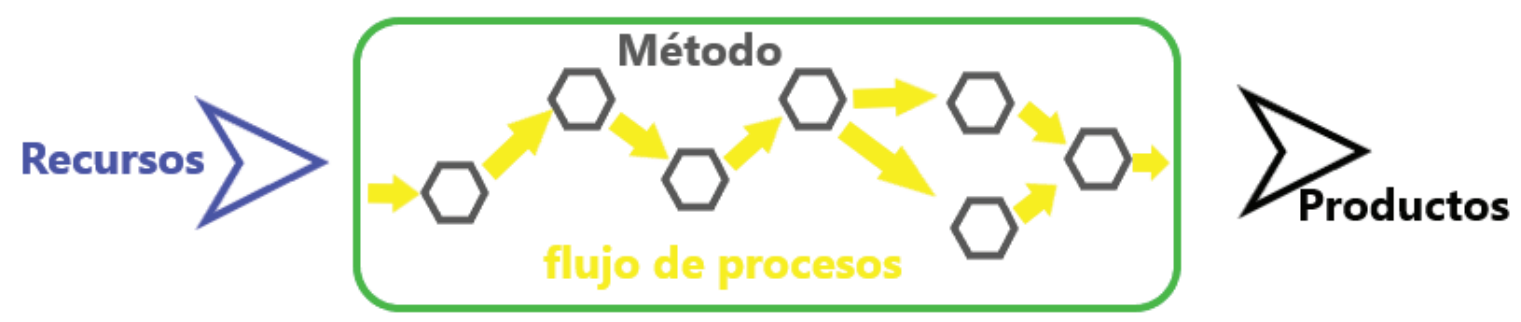

Figura 3. Elementos del proceso. Fuente: [1].

\section{El problema de la capacidad de los procesos de construcción}

La capacidad debe ser planificada de modo que los procesos sean capaces de satisfacer los requerimientos de los objetivos del proyecto de construcción. Por tanto, la capacidad de un proceso se define como el potencial máximo de producción del sistema [1].

La capacidad se puede parametrizar de acuerdo a lo siguiente:

1. Impacto significativo en la capacidad de una organización de construcción para satisfacer las demandas de un proyecto. La subcapacidad pude significar no cumplir con los plazos, gastos excesivos.

2. La relación que existe entre la capacidad y los costos de operación del sistema.

3. El costo inicial involucrado para poder proveer la capacidad requerida (capital de trabajo).

4. El compromiso de recursos que no pueden dedicarse a otros proyectos, limitando la capacidad general de la empresa constructora para tomar trabajos.

Existen diferentes capacidades por considerar en la planificación de los sistemas de producción, mencionadas en [1]:

- Capacidad de diseño: es la producción máxima que podría ser lograda o producción ideal.

- Capacidad efectiva: es la producción máxima dada una mezcla de productos, dificultades de programación, mantención de maquinarias, factores relaciona dos con la calidad, etc.

- Capacidad real: es la producción lograda, que generalmente es menor a la efectiva debido a interrupciones, defectos, falta de materiales y otros factores similares de perdida.

En función de estas capacidades, es posible establecer dos parámetros de gestión, de gran utilidad para el control de los procesos de producción y de la capacidad de ellos:

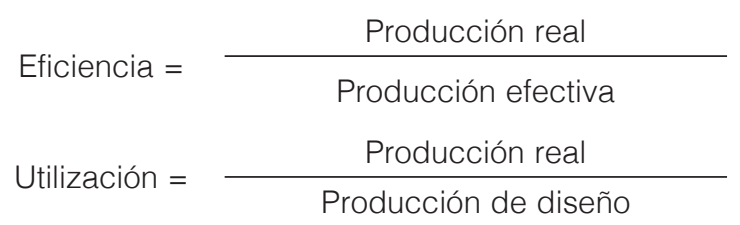


Para determinar la capacidad efectiva de un proceso constructivo, es necesario considerar los siguientes factores:

- Instalaciones productivas o bodegas, donde se incluyen la distribución, el diseño y los factores ambientales.

- Los productos que hay que producir, considerándose la calidad, constructibilidad y estandarización de su diseño.

- Los factores de los procesos, tales como la calidad y cantidad de materiales, y otros recursos de construcción disponibles.

- El factor humano y su gestión: capacitación, motivación, etc.

- Los factores asociados a la gestión del proceso de construcción, como la efectividad de la planificación y control, administración de materiales, aseguramiento y control de calidad, mantención y reparación de equipos, control de perdidas, etc.

- Factores externos, las regulaciones ambientales y de seguridad, normativa técnica, actividad sindical, etc.

\section{Tecnología}

La tecnología se define como el conjunto de procesos, herramientas, métodos, procedimientos, equipos y maquinarias que se utilizan para llevar a cabo un proceso de construcción [1]. La tecnología es un antecedente clave para la selección de procesos de construcción, ya que están íntimamente relacionados.

El uso de tecnologías innovadoras, no dominadas totalmente, puede requerir la integración de personal con capacidades especiales en ciertas funciones como la planificación, el control, el control de calidad, etc. [1]. Debe tener una clara comprensión de que las decisiones sobre tecnología involucran aspectos económicos, estratégicos, de calidad y, en general, todos los aspectos de la gestión, necesidad del cliente, estrategia de la empresa y las decisiones.

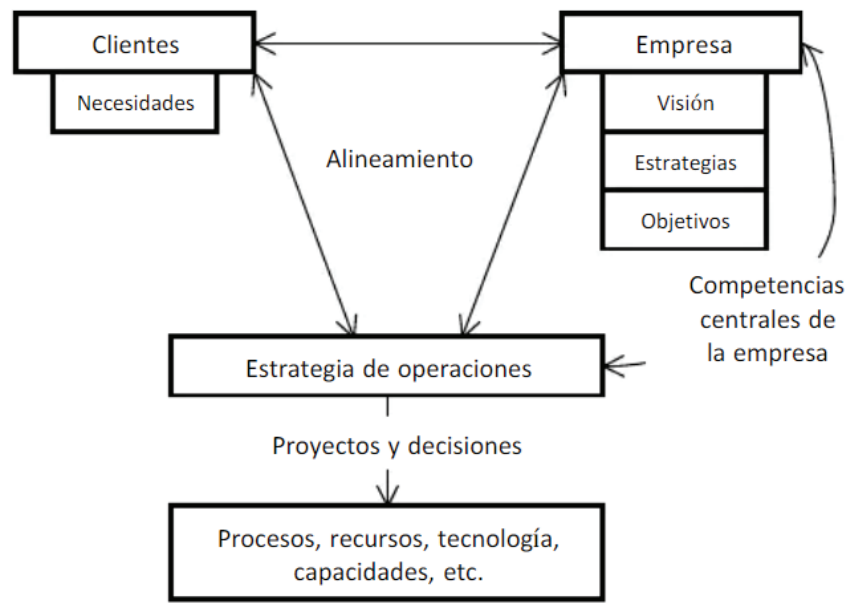

Figura 4. Estrategia de operación. Fuente: [5].

Por lo tanto, debe estudiar en profundidad los procesos de las operaciones (ver figura 4), para comprender la aplicabilidad de las tecnologías disponibles y, junto con ello, evaluar su rendimiento real. 
Planificación de la instalación de instalaciones provisionales

Instalaciones previas o provisionales podemos definirlo como un conjunto de instalaciones auxiliares necesarias, por un periodo de tiempo limitado, para la construcción y prueba de una obra. El objetivo de estás es, la maximización de la eficiencia de las operaciones para promover una alta productividad de los trabajadores y calidad del trabajo.

\section{Condiciones del entorno del proyecto}

Es fundamental analizar el entorno en que se llevara a cabo el proyecto. Para ello, es necesario evaluar los siguientes factores [1]:

- Disponibilidad de mano de obra en la zona.

- Disponibilidad de materiales y otros recursos en la zona (volúmenes máximos y disponibilidad).

- Recursos básicos (agua, electricidad, alcantarillado, etc.)

- Condiciones físicas del terreno, topografía y localización.

- Caminos de acceso al lugar de la obra, capacidad de puentes y túneles, distancias a puertos, ferrocarril, etc.

- Otros métodos de comunicación.

- Apoyos logísticos varios.

- Condiciones climáticas.

Las instalaciones provisionales podrían fluctuar entre $8 \%$ y un 15\% del costo directo de un proyecto y generalmente es cargado como gastos generales de la obra.

Principales características y tipos de instalaciones de previas y provisorias (obras provisionales)

Pero podemos definir las principales características de las instalaciones:

1. Disponibilidad de varias soluciones para una misma función.

2. Posibilidad de removerlas rápidamente al término del proyecto.

3. Posibilidad de reutilización, a un mínimo costo, para una función igual o similar en otro lugar o proyecto.

4. Facilidad para armarlas y desarmarlas, requerimiento mínimo de horas hombre.

5. Cumplimiento con estándares de seguridad y de comodidad.

6. Señalizaciones, primeros auxilios, comedores, vestuarios, servicios básicos y lockers de ser necesarios. [1]

Se pueden agrupar de acuerdo a las siguientes clasificaciones:

- Instalaciones administrativas y para el personal: incluyen las oficinas (contratistas, subcontratista, inspección, etc.) y los dormitorios, viviendas individuales, comedores, área recreativa, etc., para el personal de todos los niveles. En el cuadro 2 se muestra la relación personal directivo/subordinados por edificación y en el cuadro 3 las áreas de superficie para instalaciones provisionales. 
- Instalaciones para almacenamiento de materiales: deben estudiarse los tipos y características de cada uno: bodegas cerradas, con o sin acondicionamientos especiales, patios de aperchamiento, cubiertos y descubiertos, y, posibles bodegajes auxiliares, siempre tomando en cuenta los principales materiales.

- Instalaciones de servicio de equipos y vehículos: incluye los techos de reparación de equipos, talleres mantenimiento y reparaciones de equipos, equipos de bombas de combustibles, estacionamientos.

- Talleres auxiliares y plantas de producción en obra: triturar y clasificar piedras, concreto, premezclados, talles de moldajes, enfierradura, acero estructural, pintura, etc.

- Caminos de acceso y de circulación: problemas de drenaje, superficies de rodamiento, afectaciones por el equipo, acarreos y trasporte de equipos.

- Instalaciones básicas: Instalaciones eléctricas, agua, alcantarillado, recolecciones de agua lluvia, clasificación de desechos y reúsos.

- Cerramientos: principales para evitar el paso o acceso de personas ajenas y daños a terceros, altura mínima recomendada de 2 metros, pueden ser maderas, que se hincan en el terreno, hasta mallas metálicas de diferentes formas, lámina galvanizada lisa y ondulada, bloques y ladrillos de obra, sarán, entre otros.

Cuadro 2. Relación personal Directivo/Subordinados por edificación. Fuente: [1].

\begin{tabular}{|c|c|}
\hline Cargo & $\begin{array}{c}\text { Relación máxima subordinados } \\
\text { recomendados }\end{array}$ \\
\hline Encargado actividad & $1 / 25$ obreros \\
\hline Encargado General & $1 / 5$ Encargado Actividad \\
\hline Jefes de Obras o Maestro Obras & $1 / 4$ Encargado general \\
\hline Profesionales a cargo & $1 / 1$ Jefe de obras \\
\hline
\end{tabular}

Cuadro 3. Áreas superficie para instalaciones provisionales. Fuente: [1].

\begin{tabular}{|c|c|c|}
\hline Tipo de instalaciones provisionales & Unidad & Área/unidad (m2) \\
\hline Dormitorio & Cama & 6.50 \\
\hline Casa o Cabaña & Persona & 12.50 \\
\hline Vestidores & Persona & 0.70 \\
\hline Ducha & Persona & 0.15 \\
\hline Área recreativa & Persona & 0.85 \\
\hline Comedor & Persona & 1.40 \\
\hline Oficina & Administrativo & 6.00 \\
\hline
\end{tabular}

\section{Objetivos y recomendaciones generales para las Instalaciones de previas y provisorias}

Los objetivos específicos que la dirección eficiente de una obra debe tratar de lograr en relación a la instalación:

1. Minimizar el costo total de la instalación de provisionales.

2. Reducir al máximo el área necesaria.

3. Incrementar la productividad en obra. 
4. Establecer un grato ambiente de trabajo.

5. Obtener una buena calidad en el trabajo.

6. Aumentar la reutilización de las instalaciones.

Es importante considerar las siguientes orientaciones generales para el diseño de la instalación provisionales:

- Minimizar las distancias de viaje del personal y equipos, y de acarreo de los materiales

- Reducir interferencias de tránsito en la obra.

- Aislar las actividades contaminantes.

- Diseñar un buen sistema de drenaje en la obra.

- Estabilizar las áreas de tránsito pesado.

- Evitar la reubicación de instalaciones por falta de planificación.

- Coordinar e integrar las actividades de la instalación de faenas con el plan y el programa del proyecto.

- Estandarizar las instalaciones.

- Planificar un manejo y control eficiente de los materiales y equipos.

Se puede revisar como alcance mínimo el Capítulo XXII. Obras provisionales en el proceso de construcción, del Reglamento de construcciones - INVU. Además se deberá revisar en caso de no contar con regulaciones o plan regulador municipal, o la Ley de planificación Urbana de Costa Rica, para la delimitaciones en proyectos urbanísticos.

Planificación de corto plazo

El objetivo central de dicha planificación es lograr una alta productividad y eficiencia en la ejecución del trabajo de los siguientes objetivos operacionales:

- Planificar la producción para un horizonte de corto plazo que normalmente abarca un período entre 5 y 15 días.

- Asignar los recursos necesarios para materializar la producción deseada para el periodo.

- Fijar metas reales de producción, que aseguren el cumplimiento de los plazos.

- Evaluar y controlar el cumplimiento de las metas comparando lo realizado contra lo planificado.

- Detectar problemas que provoquen variaciones de la producción.

- Tomar acciones correctivas frente a variaciones observadas en forma rápida y eficaz.

- Delegar la autoridad necesaria a los niveles de supervisión de primera línea, dejando claramente establecida la responsabilidad asociada.

- Mejorar el control sobre la ejecución global y particular de las actividades.

- Mejorar la comunicación y retroalimentación de la ejecución del proyecto.

- Registrar la incidencia de las acciones del mandante en los resultados del contratista.

- Generar antecedentes para reclamos justos. 


\section{Características del sistema}

El sistema, presenta el siguiente esquema general.

- Planificación de la obra para la semana siguiente a la actual.

- Determinar las actividades que se desarrollarán durante el período en concordancia con el plan general de la obra. El resultado de esta etapa es el plan semanal de trabajo.

- Asignación de los trabajos a los responsables de su ejecución.

- Determinación y aseguramiento de los recursos necesario para realizar los trabajos.

- Cálculo de los resultados del trabajo semanal.

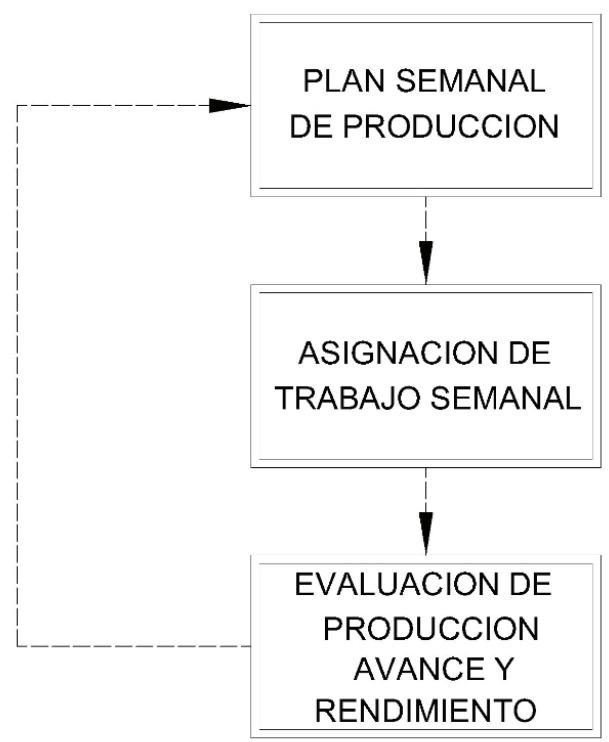

Figura 4. Sistema de Planificación a corto plazo. Fuente: [1].

Tal como se aprecia en la figura 4, el sistema presenta una metodología de planificación estructurada para el periodo semanal. Esta metodología incluye un conjunto de actividades que se deben realizar durante los días de la semana, las que se detallan en la figura 5 que se presenta a continuación.

\begin{tabular}{|c|c|c|c|c|}
\hline DIA 4 & DIA 5 & DIA 1 & DIA 2 & DIA 3 \\
\hline $\begin{array}{l}\text { PLAN SEMANAL } \\
\text { DE PRODUCCION }\end{array}$ & $\begin{array}{c}\text { VERIFICACION } \\
\text { DE LOS RECURSOS } \\
\text { A UTILIZAR }\end{array}$ & $\begin{array}{l}\text { INICIO DE LA } \\
\text { SEMANA DE } \\
\text { TRABAJO }\end{array}$ & $\begin{array}{l}\text { CALCULO DEL } \\
\text { RESULTADO } \\
\text { SEMANAL DE } \\
\text { PRODUCCION }\end{array}$ & \\
\hline & $\begin{array}{l}\text { EMISION DE LAS } \\
\text { ORDENES DE } \\
\text { TRABAJO PARA } \\
\text { JEFE DE OBRA } \\
\text { Y OPERARIOS } \\
\text { EN GENERAL }\end{array}$ & $\begin{array}{l}\text { MEDICION DE LA } \\
\text { PRODUCCION } \\
\text { SEMANAL }\end{array}$ & & \\
\hline & $\begin{array}{l}\text { TERMINO DE } \\
\text { LA SEMANA DE } \\
\text { TRABAJO }\end{array}$ & & & \\
\hline
\end{tabular}

Figura 5. Imagen plan semanal de trabajo. Fuente: [1]. 


\section{Formularios del sistema}

Estos formularios y sus objetivos, son los siguiente:

a. Plan Semanal de Producción y Control

- Registrar el control de la producción real.

- Evaluar el cumplimiento del programa.

- Ajustar la planificación para el cumplimiento de los objetivos.

b. Plan Semanal de utilización de Recursos

- Revisar la disponibilidad de recurso para cumplir el plan.

- Asignar los recursos en forma consistente con lo planificado.

- Tomar acciones correctivas frente a una subutilización de recursos o la falta de ellos.

c. Hoja de Trabajo Semanal

- Entregar la información de qué, dónde, cuánto, cuándo y cómo ejecutar la producción del período a cada supervisor de primera línea.

- Delegar la autoridad que corresponda a cada supervisor, para dar la libertad necesaria para la administración del plan.

- Asignar responsabilidades acordes con la autoridad delegada.

- Controlar el avance real y retroalimentar la planificación frente a variaciones e imprevistos detectados en terreno.

d. Evaluación de Producción, Avance y Rendimiento

- Evaluar el avance obtenido en el período considerado.

- Evaluar la producción realizada en dicho período.

- Obtener el rendimiento conseguido en el periodo.

- Retroalimentar la planificación para el siguiente período lo que se consigue con la evaluación global de la obra y del período y de las actividades en particular.

- Identificar problemas (desviaciones con respecto a lo planificado en el uso de recursos).

\section{Implementación del sistema}

Para que el sistema funcione, se requiere que todos los participantes de una obra estén dispuestos a dedicarle tiempo, a continuación, aspectos de la implementación.

- Exista compromiso de la gerencia

- El profesional de la obra, el jefe de obra, los capataces y administrativos del proyecto se comprometan y participen activamente en la planificación

- Toda la administración del proyecto asume la responsabilidad de una adecuada implementación del sistema

- Se considera que la planificación de corto plazo es una herramienta que ayuda a cumplir los objetivos de la obra en forma efectiva

- Se considera al sistema como un procedimiento habitual de la empresa 
Por las razones indicadas, es entonces muy importante estudiar cuidadosamente la implementación del sistema en una empresa u obra, de modo de asegurar que se gane la aprobación y compromiso de todos los que van a usarlo o de aquellos que sean partícipes del mismo. La figura 6 propone las etapas a seguir para una implementación exitosa.

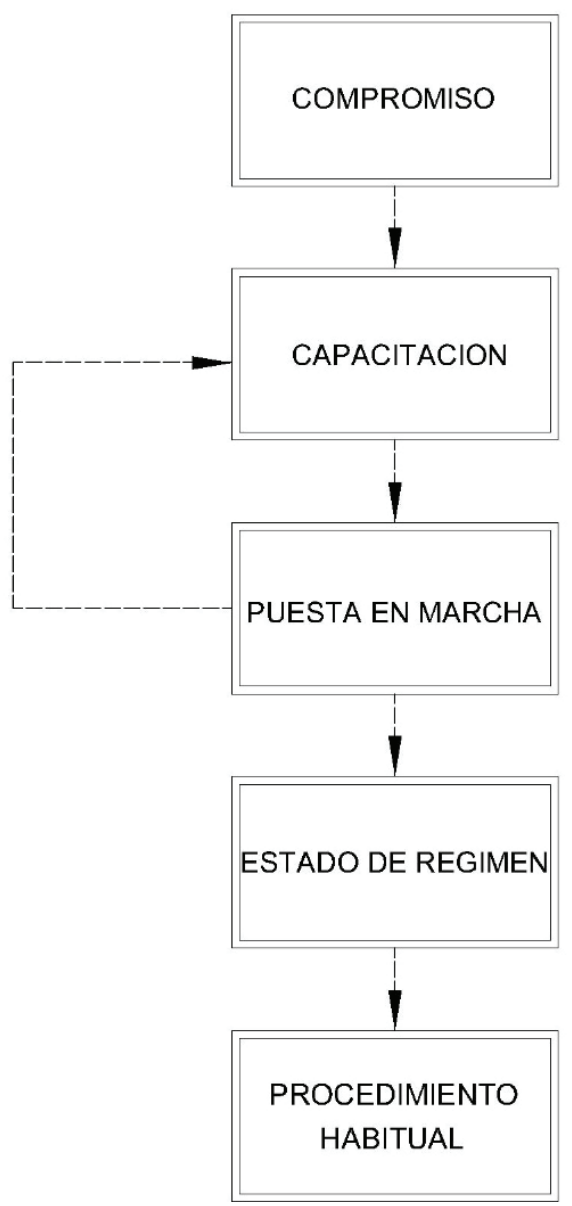

Figura 6. Etapas del plan de implementación. Fuente: [1].

Existen varias barreras que es necesario superar durante la implementación del sistema de planificación de corto plazo. Una de las primeras es aquella excusa tan concurrida en la construcción, de que los profesionales, jefes de obra, capataces y administrativos "no tienen tiempo".

Un segundo factor es la resistencia que el personal presenta ante la creación de un orden estructurado en la ejecución de la obra, producto de la planificación. Los supervisores temen perder su poder, derivado del manejo de la información de obra.

Un tercer aspecto tiene relación con la sensación de una carga adicional de trabajo que implicarla la aplicación del sistema, sin pensar en los beneficios de éste y perdiendo todo compromiso al respecto.

En la construcción es común escuchar que el personal de campo es "malo para el lápiz", indicando con esto que es difícil pedirles que entreguen la información necesaria para el funcionamiento de diferentes sistemas de control. Esta justificación es poco válida, ya que 
las personas que administran importantes recursos de una obra deben ser calificados. Si el personal no tiene la capacidad requerida, entonces es responsabilidad de la empresa el capacitarlos para que puedan responder adecuadamente a las exigencias de su trabajo.

\section{Beneficios del sistema de planificación de corto plazo}

El sistema de planificación de corto plazo aporta varios beneficios a las obras y empresas que lo utilizan. Entre los principales se destacan los siguientes:

- Permite una adecuada planificación en un período corto, mejorando la comprensión de los objetivos del proyecto.

- Ayuda al cumplimiento de los objetivos planeados en forma estructurada.

- Entrega una retroalimentación de terreno oportuna, eficaz y veraz, lo que posibilita que las proyecciones se ajusten mejor a la realidad, mejorando la toma de decisiones.

- Disminuye la ocurrencia de problemas, detectando a tiempo las distorsiones que ocurren en la obra y acelerando el aprendizaje del personal y la organización.

- Disminuye el riesgo e incertidumbre y aporta un manejo más efectivo de éstos.

- Mejora la comunicación e integración vertical y horizontal en la organización, generando una participación total de todos los estamentos directivos.

- Permite establecer prioridades consistentes con el desarrollo del proyecto.

- Aumenta la eficacia del control de terceros, tales como subcontratistas, y ayuda a la integración de los distintos intereses.

- Mejora el control de costos y plazos.

- Mejora la capacidad de respuesta del proyecto a cambios futuros, ayudando a una gestión proactiva.

- Optimiza la utilización del tiempo de la administración de la obra.

- Mejora la supervisión y control de terreno.

- Genera una base de información para el manejo de reclamos debido a acciones del mandante que han tenido consecuencias en el desarrollo de la obra.

\section{Conclusiones y/o recomendaciones}

- El proceso de planificación de la construcción debe determinar claramente cuáles son los recursos (materiales, humanos, equipos), sistemas o metodología a implementar, conociendo claramente cuál es el alcance y limitaciones de cada recurso utilizado, para poder así general el mejor producto que según la necesidad y su capacitada puede dar.

- Debe tener una clara comprensión de que las decisiones sobre tecnología involucran aspectos económicos, estratégicos, de calidad y, en general, todos los aspectos de la gestión, necesidad del cliente, estrategia de la empresa y las decisiones

- No es conveniente realizar una receta o sistema fijo de organización de obras provisionales ya que dependen del alcance y el tipo de proyecto.

- En este documento se ha presentado un breve resumen de los principales conceptos sobre temas tales como, instalaciones, reglamentos, planes, diagramas, cartas de procesos, planificación e implementación, con el objetivo de implementar estas importantes filosofías en la administración de las operaciones en construcción. 
- Todas las ideas de planificación a corto plazo presentadas pueden ser también aplicadas en forma individual a cualquier nivel de las operaciones, asegurándose siempre la calidad y el control de las mismas.

- El uso de herramientas tecnológicas, como programas de simulación de operaciones de construcción, ayudan como complemento en la toma de decisiones de los procesos constructivos y su eficacia al momento de la ejecución.

- Los diagramas de flujo, pueden ser de mucha utilidad en el diseño de la instalaciones previas y provisorias y en la distribución de las áreas de trabajo de un proyecto de construcción.

\section{Referencias}

[1] Serpell, A. (2002). ADMINISTRACIONDE OPERACIONES DE CONSTRUCCION. Santiago, Chile: ALFAOMEGA.

[2] Castillejo, W. R. (2013). Gerencia de Construcción y del Tiempo y Costo. Lima, Perú: Empresa Editora Macro E.I..R.L.

[3] Leandro Hernández, A. G. (2018). MANUAL DE BUENAS PRACTICAS PARA INCREMENTAR LA PRODUCCIÓN EN PROCESOS DE CONSTRUCCIÓN. Cartago, Costa Rica: Cámara Costarricense de la Construcción - TEC.

[4] MIDEPLAN. (2019). Guía para la Elaboración de Diagramas de Flujo. San José, Costa Rica: MIDEPLAN.

[5] Serpell Bley, A. (2015). Gestión de operación de Construccion. Obtenido de Academia.edu: https://www.academia.edu/17275294/1_La_construcci\%C3\%B3n_y_la_gesti\%C3\%B3n_de_obras_de_construcci\%C3\%B3n 